REVISTA PREVENÇÃO DE INFECÇÃO E SAÚDE (REPIS)

\title{
Cirurgia segura: recomendações adotadas antes da indução anestésica
}

Safe surgery: recommendations adopted prior to anesthetic induction

Cirugía segura: recomendaciones adoptadas antes de la inducción anestésica

Iara Maria Da Silveira ${ }^{1}$, Lorena Stephany Lopes Fernandes ${ }^{1}$, Tatiane Pereira Lopes ${ }^{1}$, Liliam Mendes de Araújo $^{2}$, Francisca Cecilia Viana Rocha ${ }^{2}$, Adélia Dalva da Silva Oliveira ${ }^{1}$

1. Centro Universitário UNINOVAFAPI, Teresina, Piauí, Brasil.

2. Universidade do Vale do Paraíba, São José dos Campos, São Paulo.

\section{RESUMO}

Objetivo: analisar a aplicação das recomendações de segurança do paciente antes da indução anestésica pelos profissionais em uma unidade de internação. Método: pesquisa quantitativa realizado na clínica ortopédica de um serviço de urgência do nordeste, Brasil. Resultados: os dados foram obtidos através da aplicação de questionários e consulta aos prontuários dos pacientes selecionados, no total de 46 sujeitos. Dos itens do formulário que também foram verificados no prontuário, todos obtiveram percentuais diferentes ao questionar o paciente. Não houve erros de sítio cirúrgico. Já os itens que devem vir preenchidos no "sing in", apenas o que diz "há acesso venoso" foi encontrado em mais de $50 \%$ dos prontuários. Conclusão: constatou-se que o protocolo de cirurgias seguras da OMS foi implementado no hospital, no entanto não há adesão satisfatória por parte dos profissionais da instituição de saúde.

Descritores: segurança do paciente; cirurgia; lista de checagem; enfermagem.

\section{ABSTRACT}

Objective: to analyze the application of patient safety recommendations prior to anesthetic induction by professionals in an inpatient unit. Method: quantitative research performed at the orthopedic clinic of an emergency service in northeast Brazil.Results: Data were obtained through the application of questionnaires and consultation of the medical records of the selected patients, in a total of 46 subjects. Of the items of the form that were also verified in the medical record, all obtained different percentages when questioning the patient. There were no surgical site errors. The items that must be filled in the "sing in", only the "venous access" is found in more than $50 \%$ of the medical records. Conclusion: it was verified that the protocol of safe surgeries of WHO was implemented in the hospital, however there is no satisfactory adherence on the part of the professionals of the health institution.

Descriptors: patient safety; surgery; check list; nursing.

\section{RESUMÉN}

Objetivo: analizar la aplicación de las recomendaciones de seguridad del paciente antes de la inducción anestésica por los profesionales en una unidad de internación. Método: investigación cuantitativa realizada en la clínica ortopédica de un servicio de urgencia del nordeste, Brasil. Resultados: los datos fueron obtenidos a través de la aplicación de cuestionarios y consulta a los prontuarios de los pacientes seleccionados, en el total de 46 sujetos. De los elementos del formulario que también fueron verificados en el prontuario, todos obtuvieron porcentajes diferentes al cuestionar al paciente. No hubo errores de sitio quirúrgico. Los elementos que deben cumplirse en el "sing in", sólo lo que dice "hay acceso venoso" fue encontrado en más del $50 \%$ de los prontuarios. Conclusión: se constató que el protocolo de cirugías seguras de la OMS fue implementado en el hospital, sin embargo no hay adhesión satisfactoria por parte de los profesionales de la institución de salud.

Descriptores: seguridad del paciente; cirugía; lista de comprobación; enfermería.

Como citar este artigo:

Silveira IM, Fernandes LSL, Lopes TP, Araújo LM, Rocha FCV, Oliveira ADS. Cirurgia segura: recomendações adotadas antes da indução anestésica. Rev Pre Infec e Saúde[Internet]. 2018;4:7285. Available from: http://www.ojs.ufpi.br/index.php/nupcis/article/view/7285 DOI: https://doi.org/10.26694/repis.v4i0.7285 


\section{INTRODUÇÃO}

A preocupação com a qualidade é inerente ao ser humano. Esta evolui com passar dos anos, de formas variadas, com esforços para que não haja a imperfeição. A assistência prestada aos pacientes deve ser de forma integral e livre de danos $^{1}$. Assim, o profissional implementa o que estabelece a segurança do paciente que consiste, não somente em prevenir, mas em melhorar os resultados adversos oriundos do atendimento médico-hospitalar e também no domiciliar².

Buscando aprimorar a assistência cirúrgica a organização mundial de saúde (OMS) estabeleceu uma ferramenta para assegurar que as equipes de saúde dos hospitais que realizam cirurgias, sigam de maneira consistente as medidas de segurança, reforçando as práticas de segurança e promovendo melhor comunicação, consequentemente, aumentando a segurança dos procedimentos cirúrgicos. Todavia, a lista de verificação de segurança cirúrgica (checklist) ainda sofre constantes recomendações de novas modificações e adaptações para torna-la mais ampla ${ }^{3}$.

Segundo a Organização Mundial de Saúde (OMS), no ano de 2008, foram realizadas cerca de 234 milhões de intervenções cirúrgicas, com média de uma cirurgia a cada 25 pessoas. Destas, estima-se que ocorreram sete milhões de complicações e dois milhões de mortes, sendo que a metade dessas mortes e complicações eram evitáveis, o que sugere que, mesmo com o intuito de salvar vidas, as falhas de segurança nos procedimentos cirúrgicos podem causar danos importantes ao paciente, Rev Pre Infec e Saúde.2018;4:7285 como a invalidez ou a morte ${ }^{4-5}$.

Um estudo exploratório, descritivo com abordagem quantitativa, realizado em um Centro Cirúrgico de uma instituição privada de São Paulo, com profissionais, por meio da aplicação de um questionário estruturado com o objetivo de verificar a opinião da equipe cirúrgica sobre a realização do time out e identificar as facilidades e dificuldades para sua aplicação, constatou que todos os participantes conhecem o time out e a maioria reconhece sua eficácia e acredita que a aplicação traz segurança para o cliente e para a própria equipe. Os fatores que facilitam a aplicação do time out são: colaboração da equipe, conhecimento, clareza e objetividade do protocolo; os que dificultam são: a falta de colaboração da equipe, protocolo longo e repetitivo e atrasos dos médicos e das cirurgias. A implementação do time out é um desafio para a instituição e para a equipe, porém o protocolo contribui para redução de riscos e de eventos adversos, proporcionando segurança ao cliente $^{6}$.

Um estudo realizado com voluntários, ortopedista, que participavam de um evento de saúde no ano de 2012 através da aplicação de um questionário sobre o uso do protocolo de cirurgia segura da OMS, constatou que cerca de $40,8 \%$, relatou ter presenciado erros de sítio cirúrgico, destes $25,6 \%$ apontavam como umas das causas a falta de comunicação efetiva entre os profissionais ${ }^{7}$.

A relevância deste estudo se dar devido a esta temática ser de relevância mundial e vim sendo abordada na atualidade com maior frequência, dada sua importância e a 
Silveira IM, et al

ocorrência de danos aos pacientes resultantes de práticas inseguras. garantir um cuidado seguro é considerada um elemento essencial para a efetividade do cuidado, sendo importante não somente para os pacientes, mas para família, profissionais e gestores.

neste contexto, a redução de danos ao paciente cirúrgico ocupa um lugar de destaque, visto que garantir uma cirurgia segura é uma das metas da Organização Mundial de Saúde. Dessa maneira, seguir o protocolo para garantir uma melhor segurança dos pacientes submetidos à cirurgia será fundamental para evitar eventos adversos ou até mesmo a morte. 0 presente estudo contribui para propor ações que assegurem uma assistência livre de danos aos clientes cirúrgicos no âmbito hospitalar.

Diante desta problemática, é necessária a adesão de todos os envolvidos para aplicar o checklist, com o propósito de minimizar os riscos e danos ao paciente que irá submeter-se a algum tipo de cirurgia. portanto está pesquisa tem por objetivo analisar a aplicação das recomendações de segurança do paciente antes da indução anestésica pelos profissionais em uma unidade de internação.

\section{MÉTODOS}

Este estudo foi submetido ao comité de ética e pesquisa da Fundação Hospitalar de Teresina para autorização da realização do estudo, posteriormente ao CEP do UNINOVAFAPI, que é um órgão colegiado de natureza técnicocientífica, vinculado ao CONEP, recebendo parecer favorável e aprovado pelo número 1.968.876, em 16 de março de 2017.
Recomendações adotadas para indução anestésica

Versa sobre uma pesquisa quantitativa descritiva realizada na Clínica Ortopédica de um serviço de Urgência do Nordeste, Brasil, no segundo bimestre de 2017. O tipo de amostragem foi não probabilístico, por acessibilidade ou conveniência, pois, segundo ${ }^{8}$, está necessita apenas de critérios determinados pelo pesquisador, onde considera os elementos acessíveis e que possam reproduzir o universo com os resultados obtidos, não fazendo uso dos fundamentos estatísticos e matemáticos.

compõem a amostragem 46 pessoas, dentre elas homens e mulheres que tenham ou perpassaram a maior idade e tenham sido submetidos à primeira intervenção cirúrgica e foram excluídos do estudo os pacientes internados que já se submeteram a um ou mais procedimentos cirúrgicos e que estiveram retornando para uma nova cirurgia que caracterize uma complementação da anterior, aqueles legalmente incapazes e os que apresentem algum transtorno mental que os tornem inaptos a compreender os objetivos do estudo.

Como instrumento para produção de dados foi utilizado um formulário composto com as seguintes questões: caracterização sóciodemográfica e econômica dos participantes (idade, sexo, situação conjugal, renda, escolaridade); dados referentes à internação e procedimento cirúrgico e checklist conforme as recomendações da OMS antes da indução anestésica. A obtenção dos dados se deu através da aplicação de questionários aplicados aqueles pacientes que atenderam aos critérios préestabelecidos estes foram respaldados eticamente assinando ao Termo de 
Silveira IM, et al

Consentimento Livre e Esclarecido (TCLE) e pela verificação da aplicabilidade do checklist pelos profissionais no que se refere às recomendações da OMS, antes da indução anestésica, através da análise do preenchimento deste instrumento nos prontuários dos pacientes selecionados. Como instrumento para produção de dados foi utilizado um formulário composto com as seguintes questões: caracterização sócio-demográfica e econômica dos participantes (idade, sexo, situação conjugal, renda, escolaridade e etnia); dados referentes à internação e procedimento cirúrgico e checklist, conforme as recomendações da OMS antes da indução anestésica.

Por ser um estudo analítico descritivo, foram feitos cálculos percentuais utilizando-se o Microsoft Office Excel por se tratar de um Software estatístico que possibilita analisar os dados coletados com eficácia e ser de fácil manuseio. Após processados e consolidados, a discussão foi feita com base nas publicações científicas sobre o tema.

\section{RESULTADOS}

Os dados serão apresentados em duas tabelas e
Recomendações adotadas para indução anestésica dois gráficos. A Tabela 1 traz a distribuição da amostra quanto à caracterização sociodemográfica e econômica; Tabela 2: procedimentos antes da indução anestésica (conferência junto às anotações no prontuário); Gráfico 1: distribuição da amostra segundo Sítio Cirúrgico e o Gráfico 2 traz a distribuição da amostra segundo informações sobre o procedimento antes da indução anestésica.

A Tabela 1 apresenta os dados sobre as características dos pacientes quanto à idade; esta variou entre 18 e 51 anos, tendo como predominante a faixa etária entre 31-35 anos (22\%), seguida do percentual $18 \%$, cuja faixa etária abrange de 21-25 anos. Quanto ao sexo, o percentual predominante dentre os 46 entrevistados foi o masculino, com76\%.

Em relação à variável escolaridade, $48 \%$ dos entrevistados possuíam de 4 a 7 anos de estudo. Quando ao estado conjugal dos pacientes, 29 (63\%) eram casados ou em união estável. Os dados relativos à renda revelaram que $65 \%$ dos pacientes ganham entre 1 a 2 salários mínimos; a maioria considera-se de etnia parda (63\%).

Tabela 1 - Distribuição da amostra $(n=46)$ segundo características sociodemográficas e econômicas, Teresina, PI, 2017.

\begin{tabular}{lll}
\hline Variáveis & $\mathbf{N}$ & $\%$ \\
\hline FaixaEtária & & \\
$18-20$ & 05 & 11 \\
$21-25$ & 08 & 18 \\
$26-30$ & 04 & 9 \\
$31-35$ & 10 & 22 \\
$36-40$ & 05 & 11 \\
$41-45$ & 05 & 11 \\
$46-50$ & 02 & 5 \\
$51-$ mais & 06 & 13
\end{tabular}




\begin{tabular}{|c|c|c|}
\hline & & (continuação) \\
\hline Variáveis & $\mathbf{N}$ & $\%$ \\
\hline \multicolumn{3}{|l|}{ Sexo } \\
\hline Masculino & 35 & 76 \\
\hline Feminino & 11 & 24 \\
\hline \multicolumn{3}{|l|}{ Escolaridade } \\
\hline Nenhum & 01 & 2 \\
\hline 1 a 3 & 01 & 2 \\
\hline 4 a 7 & 22 & 48 \\
\hline 7 a 11 & 20 & 44 \\
\hline 12 ou mais & 02 & 4 \\
\hline \multicolumn{3}{|l|}{ Situação Conjugal } \\
\hline Solteira (o) & 15 & 33 \\
\hline Casada (a)/uniãoestável & 29 & 63 \\
\hline Viúva (o) & - & - \\
\hline Separada (o) judicialmente/divorciada & 02 & 4 \\
\hline Outros & - & - \\
\hline \multicolumn{3}{|l|}{ Renda } \\
\hline Nenhum & 07 & 15 \\
\hline Menorque 1 & 06 & 13 \\
\hline 1 a 2 & 30 & 65 \\
\hline 3 a 5 & 03 & 7 \\
\hline 6 a 9 & - & - \\
\hline$>10$ & - & - \\
\hline \multicolumn{3}{|l|}{ Etnia } \\
\hline Branca & 05 & 11 \\
\hline Preta & 09 & 20 \\
\hline Amarela & 03 & 6 \\
\hline Parda & 29 & 63 \\
\hline Indígena & - & - \\
\hline
\end{tabular}

De acordo com o Gráfico 1, quanto ao sítio cirúrgico, $57 \%$ tiveram fraturas nos MMII (membros inferiores) e os outros $43 \%$ nos MMSS (membros superiores). Constam nestes dados os pacientes com fraturas expostas e fechadas de nervos até ossos maiores, como o fêmur. Estes $67 \%$ foram causados por acidente de motocicleta e os demais por acidente de trabalho ou queda da própria altura. Segundo ${ }^{9}$, queda é delineada como "um deslocamento não intencional do corpo a um nível inferior em relação à posição inicial, com incapacidade de correção em tempo hábil, determinado por circunstâncias multifatoriais, comprometendo a estabilidade". Esse fenômeno é atribuído tanto as causas internas quanto as externas, denominadas respectivamente de intrínsecas e extrínsecas. Temos também outros fatores, totalizando um percentual de $11 \%$ cada. 
Gráfico 1 - Distribuição da amostra (n=46) segundo sítio cirúrgico, Teresina, PI, 2017.

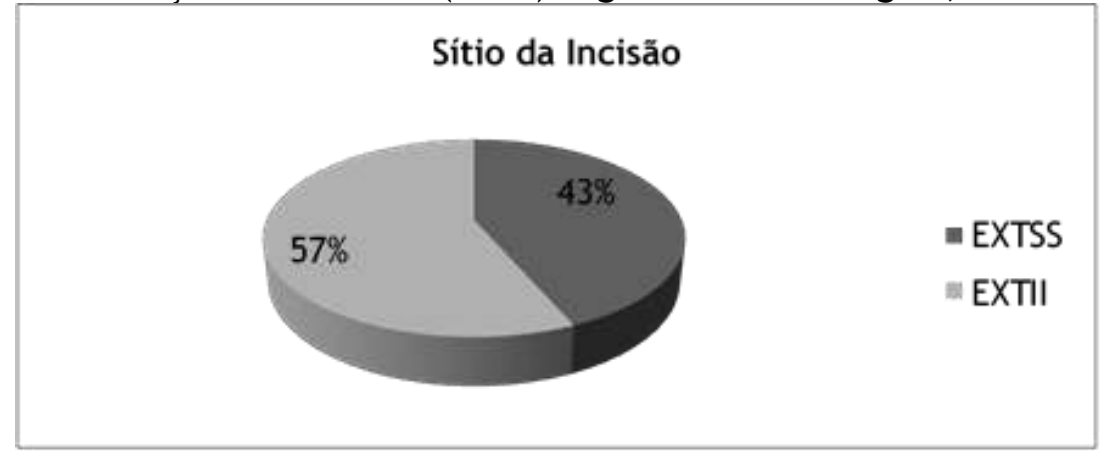

Legenda: EXTSS - extremidades superiores; EXTII - extremidades inferiores.

O Gráfico 2 mostra os dados referentes aos pacientes que receberam informações antes da indução anestésica; um percentual de 59\% recebeu orientações quanto à cirurgia e $41 \%$ não foram orientados. 0 profissional mais citado pelos pacientes foi o médico (43\%), seguido do enfermeiro (11\%) e do anestesiologista (2\%).

De acordo com os dados da amostra, somente $11 \%$ tiveram a cirurgia cancelada. A falta de material específico para a realização da cirurgia foi citada como um dos motivos para o cancelamento do procedimento cirúrgico.

Gráfico 2 - Distribuição da amostra $(n=46)$ segundo informações sobre o procedimento antes da indução anestésica, Teresina, PI, 2017.

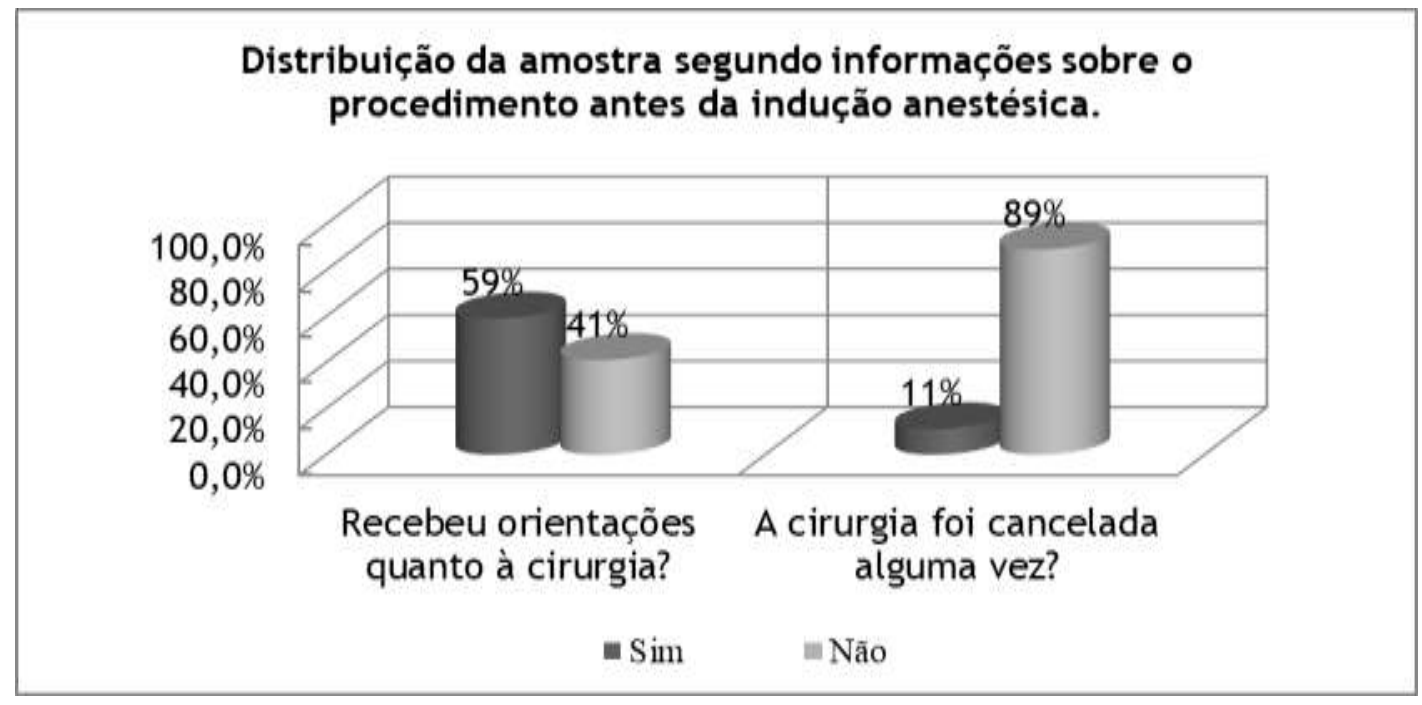

A tabela 2 mostra informações relativas a dois momentos diferentes: primeiro, os dados obtidos por meio da consulta aos prontuários conforme a folha do time out, em que constam as informações que devem ser verificadas conforme as recomendações da OMS antes da Rev Pre Infec e Saúde.2018;4:7285 indução anestésica e o segundo durante entrevista com o paciente. Quando questionados se, ao entrar no CC (Centro Cirúrgico), possuíam pulseira de identificação no braço, 57\% responderam que sim; a consulta ao prontuário mostrou que esta informação 
Silveira IM, et al

estava presente em78\%. $87 \%$ dos pacientes afirmaram conhecer o local da cirurgia a ser feita, percentual (83\%) que se confirmou na consulta ao prontuário; já ao verificar se o sítio cirúrgico do lado certo está sendo registrado, $100 \%$ demonstram que sim.

Quanto ao procedimento a ser realizado, apenas $76 \%$ dos pacientes afirmaram ter conhecimento, mas em $80 \%$ dos prontuários ele estava descrito. 0 consentimento informado foi assinado por $50 \%$ dos pacientes e confirmado nos prontuários em $54 \%$ deles e $46 \%$ não foram assinados.

A checagem do equipamento anestésico não constava em $61 \%$ dos prontuários dos pacientes entrevistados e o item oxímetro de pulso instalado e funcionando teve um percentual parecido, com 52\%.

No quesito alergias, $89 \%$ dos pacientes entrevistados confirmaram terem sido
Recomendações adotadas para indução anestésica

questionados sobre possuírem algum tipo de alergia. Porém, quando verificado no prontuário esse percentual, caiu para $76 \%$. Quanto à existência de risco de via aérea difícil/broncoaspiração, as informações constavam somente em apenas $13 \%$ dos prontuários.

Sobre o risco de perda sanguínea, $17 \%$ dos entrevistados tinham em seus prontuários a confirmação e $83 \%$ deles não foram preenchidos. Com relação ao planejamento de reposição, em 13\% havia confirmação no prontuário e nos outros $87 \%$ não. 0 item acesso venoso teve um percentual de $80 \%$ e em $20 \%$ dos prontuários não havia está informação. Já quanto ao carro de anestesia completo e liberação para uso, constava em apenas $9 \%$ dos prontuários pesquisados e em $91 \%$ não havia nenhum registro quanto a essa informação.

Tabela 2 - Distribuição da amostra $(n=46)$ segundo procedimentos realizados antes da indução anestésica, Teresina, PI, 2017.

\begin{tabular}{|c|c|c|c|c|c|c|c|c|}
\hline \multirow{3}{*}{ Variáveis } & \multicolumn{8}{|c|}{ Conhecimento } \\
\hline & \multicolumn{4}{|c|}{ Prontuário } & \multicolumn{4}{|c|}{ Confirmadopelopaciente } \\
\hline & \multicolumn{2}{|c|}{ Sim } & \multicolumn{2}{|c|}{ Não } & \multicolumn{2}{|c|}{ Sim } & \multicolumn{2}{|c|}{ Não } \\
\hline Identificação do paciente & 36 & 78 & 10 & 22 & 26 & 57 & 20 & 43 \\
\hline Local da cirurgia a ser feita & 40 & 87 & 06 & 13 & 38 & 83 & 08 & 17 \\
\hline Procedimento a serrealizado & 37 & 80 & 09 & 20 & 35 & 76 & 11 & 24 \\
\hline Consentimento informado realizado & 23 & 50 & 23 & 50 & 25 & 54 & 21 & 46 \\
\hline Alergias do paciente & 35 & 76 & 11 & 24 & 41 & 89 & 05 & 11 \\
\hline Sítio incisão do lado certo & 46 & 100 & - & & 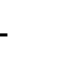 & & - & - \\
\hline Conferir os equipamentos de anestesia & 18 & 39 & 28 & 61 & - & 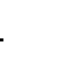 & - & - \\
\hline
\end{tabular}


Oxímetro de pulso instalado e funcionando

Existência de risco de via aérea difícil/broncoaspiração

Risco de perda sanguínea $>500 \mathrm{ml}$

Há acesso venoso

Há planejamento para reposição

Informações quanto ao carro de anestesia completo e liberação para uso

\begin{tabular}{l|l|l|l|}
22 & 48 & 24 & 52 \\
06 & 13 & 40 & 87 \\
08 & 17 & 38 & 83 \\
37 & 80 & 09 & 20 \\
06 & 13 & 40 & 87 \\
04 & 9 & 42 & 91
\end{tabular}

\section{DISCUSSÃO}

A característica social e demográfico mais constatado foi na faixa etária de 20 a 39 anos, escolaridade entre 9 e 11 anos de estudo e sexo masculino ${ }^{10-11}$ foram alguns dos estudos encontrados com perfil que se assemelha ao desta amostra. A situação conjugal casado (a)/união estável predomina. A renda e a etnia são semelhantes às da realidade da população da região. No que diz respeito à escolaridade, demonstrou-se uma baixa escolaridade dos sujeitos assistidos na clínica estudada, confirmando os achados da literatura ${ }^{12}$.

As fraturas são responsáveis por cerca de $38 \%$ das internações por causas externas ${ }^{13}$. Neste estudo, no que se refere às causas das fraturas, estas se assemelham às encontradas na literatura, sendo a motocicleta uma das maiores responsáveis, presente em mais da metade dos casos notificados $(50,9 \%)^{14}$. Os autores relatam que, no Brasil, o planejamento urbano não acompanhou a evolução da quantidade de transportes terrestres, tornando Rev Pre Infec e Saúde.2018;4:7285 as pessoas suscetíveis a eventos como esses.

0 processo que envolve o momento préanestésico deve ser realizado através do primeiro momento, "Sign in", do Checklist desenvolvido pela OMS, já citado nesta pesquisa, criado com o intuito de auxiliar as equipes operatórias na redução das ocorrências de danos ao cliente. Este deve ser consumado com o acompanhamento impreterível do enfermeiro e anestesiologista, considerando-se as recomendações de ações que serão discutidas a seguir. No entanto, nesta pesquisa, ao serem questionados quanto ao recebimento de orientações sobre o procedimento cirúrgico e qual profissional seria responsável pela mesma, o profissional médico foi citado por $77 \%$ dos pacientes.

Embora as anotações tenham que ser realizadas em todos os tipos de cirurgias, desde uma simples reparação, até uma mais complexantes, dentre eles a feitura do ato cirúrgico, evitam um erro muitas vezes irreparável mais tarde e, mesmo assim, de acordo com os dados desta pesquisa, boa parte 
(87\%) possui este registro no prontuário. Porém, não é possível afirmar que estejam ocorrendo erros de sitio cirúrgicos, pois ao verificar com os participantes e em seus prontuários, 100\% das cirurgias foram realizadas no local certo, isto pode ser respaldado devido ao fato de serem pacientes que chegam no setor já com fraturas expostas e os de fraturas fechadas com os membros imobilizados por uma tala, evidenciando o local da fratura.

Em $80 \%$ dos prontuários analisados havia a descrição do procedimento a ser realizado, no entanto, apenas $76 \%$ dos pacientes entrevistados afirmaram ter algum conhecimento sobre o assunto, embora seja um direito garantido pela Portaria $n^{\circ} 1.820$, de 13 de agosto de $2009^{6}$. É direito do paciente ser previamente informado sobre as possíveis mudanças, sejam elas temporárias ou não, devido ao ato cirúrgico ${ }^{15}$.

Deve-se respeitar a vontade do paciente, permitindo que o mesmo participe ativamente do seu processo terapêutico ${ }^{16}$; porém, a realidade encontrada nesta pesquisa é que apenas em metade (50\%) dos prontuários constava o Termo de Consentimento Informado (TCl) e em muitos casos havia apenas a assinatura do médico e paciente/acompanhante, sem preenchimento das informações e, ao questionar o paciente, $4 \%$ a mais informaram ter assinado; estes resultados podem estar relacionados à falta de informação ao paciente/acompanhante por parte do profissional.

Em um estudo para identificar o entendimento do paciente cirúrgico acerca do $\mathrm{TCl}$, evidenciaram que os pacientes, embora não saibam o seu objetivo, o consideraram relevante. Constataram assim que as informações repassadas aos sujeitos da pesquisa antes da assinarem 0 termo não foram suficientes para que ficasse claro o seu objetivo $^{17}$.

A ausência de verificação do equipamento anestésico antes da sua utilização vem sendo agregada a expansão do risco de morbidade e mortalidade pós-operatórias. Dos itens da lista de verificação, este foi um dos que teve um percentual de adesão por parte dos profissionais abaixo de $50 \%$, seguido por oxímetro de pulso instalado e funcionando (48\%), risco de perda sanguínea > 500ml (17\%), planejamento para reposição e existência de risco de via aérea difícil/broncoaspiração com o mesmo percentual (13\%) e informações quanto ao carro de anestesia completo e liberação para uso (9\%).

\section{CONCLUSÃO}

Considerando os dados elucidados no discorrer deste estudo, observamos que o protocolo de cirurgias seguras da OMS foi implementado no hospital, no entanto não há adesão satisfatória por parte dos profissionais da instituição de saúde.

Conclui-se também que a instituição, ao implantar os protocolos recomendados pela OMS, também deve treinar e supervisionar todo o processo, tendo em vista a necessidade de acompanhar e garantir a adesão por parte dos profissionais, para assim garantir a segurança na assistência. No que se refere aos procedimentos cirúrgicos, esta somente é garantida quando os três momentos do 
Silveira IM, et al

protocolo forem seguidos corretamente, ou seja, constar no prontuário, ser preenchido por completo e o comprometimento da equipe.

Por fim, destaca-se que o processo de implantação do checklist para Cirurgia Segura é um desafio, não somente para instituições de saúde, gestores, gerentes, mas também para os profissionais de saúde. Dessa maneira, faz-se necessário maior investimento na divulgação de informações sobre o tema, mais estudos e um maior envolvimento por parte dos envolvidos, destacando neste contexto a importante participação do paciente em relação a sua própria segurança.

\section{REFERÊNCIAS}

1. Lima CA, Faria JS, Machado APN, Gonçalves RPF, Teixeira MG, Oliveira RS, et al. Hospital risk management: a focus on the quality and the patient safety. Rev. Eletrônica Gestão Saúde. 2014; 5(Spe): 2862-76. Disponível em: http://dx.doi.org/10.18673/gs.v5iespecial.1383 22.

2. Vincent C. Risk, safety and the dark side of quality. BMJ [Internet] 1997; 314, 7097, 1775- 6 [acesso em 28 out 2016]. Disponível em: https://www.ncbi.nlm.nih.gov/pmc/articles/PM C2126912/

3. Freitas MR. Avaliação da adesão ao checklist de cirurgia segura da OMS em cirurgias urológicas e ginecológicas, em dois hospitais de ensino de Natal, Rio Grande do Norte, Brasil. Cad. Saúde Pública [Internet] 2014; 30;1; 1337 48 [acesso em 31 de out 2016]. Disponível em:http://www.scielosp.org/scielo.php?script=s ci_arttext\&pid=S0102-
Recomendações adotadas para indução anestésica 311X2014000100137\&lng=en.http://dx.doi.org/1 0.1590/0102-311X00184612.5

4. Organização Mundial da Saúde (OMS). Segundo desafio global para a segurança do paciente: Manual - cirurgias seguras salvam vidas. Rio de Janeiro: Organização Pan-Americana da Saúde, Ministério da Saúde, Agência Nacional de Vigilância Sanitária [Internet] 2009 [acesso 16 set 2016]. Disponível em:http://bvsms.saude.gov.br/bvs/publicacoes/ seguranca_paciente_cirurgia_salva_manual.pdf 5. Ferraz EM. A cirurgia segura: uma exigência do século XXI. Rev. Col. Bras. Cir. [Internet] 2009; 36, 4,281-2 [acesso 16 set 2016].

6. Martins GS, Carvalho R. Realização do time out pela equipe cirúrgica: facilidades e dificuldades. Rev. SOBECC, São Paulo [Internet] 2014; $19, \quad 1, \quad 18-25$. /site_sobecc_v19n1/04_sobecc_v19n1.pdf

7. Motta filho GRM et al. Protocolo de Cirurgia Segura da OMS: 0 grau de conhecimento dos ortopedistas brasileiros. Rev. Bras. Ortop. [Internet] 2013; 48, 6, 554-562 [acesso 16 set 2016]. Disponível em: http://www.scielo.br/pdf/rbort/v48n6/pt_0102 -3616-rbort-48-06-00554.pdf

8. Freitas R. et al. Cuidado de enfermagem para prevenção de quedas em idosos: proposta para ação. Rev. Bras. Enferm., Brasília [Internet] 2011, 64(3): 478-85 [acesso 27 maio 2017]. Disponível em: http://www.scielo.br/pdf/reben/v64n3/v64n3a 11.pdf

9. Gil AC. Como elaborar projetos de pesquisa.São Paulo: Atlas [Internet] 2008; 5.

10. Legay LF et al. Acidentes de transporte envolvendo motocicletas: perfil epidemiológico 
das vítimas de três capitais de estados 2017].

Disponível

em:

brasileiros, 2007. Epidemiol. Serv. Saúde, Brasília [Internet] 2012; 21(2):283-292 [acesso 22 maio2017].

Disponível em:http://scielo.iec.pa.gov.br/scielo.php?script =sci_arttext\&pid=S1679-49742012000200011

11. Noronha CKC et al. Ocorrência de óbitos por acidentes de motocicletas em Teresina, Estado do Piauí, Brasil. Rev. Pan-Amaz. Saude [Internet] 2011; 2(4):11-16.

12. Belon AP et al. Atendimentos de emergência a vítimas de violências e acidentes: diferenças no perfil epidemiológico entre o setor público e o privado. VIVA - Campinas/SP. Ciência \& Saúde Coletiva [Internet] 2012; 17(9):2279-2290 [acesso 9 maio 2017]. Disponível em: http://www.scielo.br/pdf/csc/v17n9/a10v17n9. pdf

13. Minayo MCS, Deslaandes S F. Análise diagnóstica. Rio de Janeiro: Ed Fio- cruz; 2007.

14. Cavalcante AKCB. Perfil dos acidentes de trânsito atendidos por serviço pré-hospitalar móvel. Revista Baiana de Enfermagem, Salvador [Internet] 2015; 29, 2, 135-145 [acesso 09 mai https://portalseer.ufba.br/index.php/enfermag em/article/viewFile/12656/pdf_125

15. Ferraz AF. Análise da comunicação enfermeira-paciente em hospital geral [dissertação]. São Paulo (SP): Escola de Enfermagem, Universidade de São Paulo, 1993.

16. Clotet J. O consentimento informado nos comitês de ética em pesquisa e na prática médica: conceituação, origens e atualidade. Rev. Bioét. [Internet] 2009; 3(1):51-9 [acesso 06 out 2016]. Disponível em: http://www.pucrs.br/bioetica/cont/clotet/ocon sentimento.pdf

17. Melendo MP et al. Termo de consentimento informado: entendimento do paciente cirúrgico. Acta Paul. Enferm. [internet] 2016; 29(3):291-7 [acesso 20 mai 2017]. Disponível em:http://www.scielo.br/pdf/ape/v29n3/19820194-ape-29-03-0291.pdf

\section{Submetido: 2018-06-20}

Aceito: 2018-09-02

Publicado: 2018-10-01

\section{COLABORAÇÕES}

Silveira IM, Fernandes LSL e Lopes TP declaram que contribuíram com a concepção do estudo, análise, interpretação dos dados, redação do artigo, revisão crítica relevante do conteúdo. Araújo LM, Rocha FCV e Oliveira ADS colaboraram nas etapas de revisão crítica relevante do conteúdo intelectual. Todos os autores concordam e se responsabilizam pela versão final a ser publicada.

\section{CONFLITOS DE INTERESSE}

Não há conflitos de interesse a declarar.

\section{CORRESPONDÊNCIA}

Adélia Dalva da Silva Oliveira.

Centro Universitário UNINOVAFAPI.

Rua Vitórino Orthiges Fernandes, 6123 - Uruguai, Teresina - PI, 64073-505.

Email: aoliveira@uninovafapi.edu.br 